EPJ Web of Conferences 53, 04007 (2013)

DOI: $10.1051 /$ epjconf/20135304007

(C) Owned by the authors, published by EDP Sciences, 2013

\title{
Measurements of the longitudinal shower development with the Pierre Auger Observatory
}

\author{
V. de Souza ${ }^{1}$ for the Pierre Auger Collaboration ${ }^{2, a}$ \\ 1 Universidade de São Paulo, Instituto de Física de São Carlos, SP, Brazil \\ 2 Pierre Auger Observatory, Av. San Martín Norte 304, (5613) Malargüe, Argentina \\ http://www.auger.org/archive/authors_2012_06.html
}

\begin{abstract}
In this work we explain how the Pierre Auger Observatory measures and reconstructs the longitudinal development of air showers. The measurement of the energy deposit in the atmosphere by the detection of the emitted fluorescence light is going to be briefly reviewed and the reconstruction procedure is going to be explained in detail. The two main outputs of this analysis are: a) the depth at which the shower reaches its maximum $\left(X_{\max }\right)$ and b) the shower energy. In this work we concentrate on the analysis techniques developed with the aim to evaluate the unbiased $X_{\max }$ distributions. We show how using measured events it is possible to estimate the range of the true $X_{\max }$ distribution. Then we select events with geometries that allow an unbiased measurement of $X_{\max }$ anywhere along its estimated range. The analysis procedure explained here is a fundamental step in the measurements of cosmic ray mass composition and proton-air cross section.
\end{abstract}

\section{INTRODUCTION}

The Pierre Auger Observatory measures cosmic ray showers using two techniques: water Cherenkov detectors and fluorescence telescopes. Water Cherenkov detectors measure the particles in the shower when they reach the ground. Fluorescence telescopes detect the fluorescence light produced in the atmosphere due to the passage of the particles in air. The telescopes measure the evolution of the shower along the atmosphere.

In this paper we review the detection and data analysis of the fluorescence telescopes measurement. Special attention is going to be given to the procedure adopted to calculate the depth in which the shower reaches its maximum $\left(X_{\max }\right)$.

$X_{\max }$ is the most used parameter in composition studies. The Pierre Auger Observatory has published the evolution of $\left\langle X_{\max }\right\rangle$ (mean of the $X_{\max }$ distribution) and $\operatorname{RMS}\left(X_{\max }\right)$ (RMS of the $X_{\max }$ distribution) as a function of energy [1]. In this paper we review the details of the data analysis which lead to the determination of an unbiased sample of $X_{\max }$ that can be compared directly to Monte Carlo predictions.

In a different study, the tail of the $X_{\max }$ distribution was used to measure the proton-air cross section as shown in $[2,3]$. The data analysis procedure used in this study is very similar to the one adopted in the $\left\langle X_{\max }\right\rangle$ and $\operatorname{RMS}\left(X_{\max }\right)$ measurements.

We start reviewing the fluorescence telescope characteristics in section 2, we discuss the measurements in section 3, the data analysis in section 4 and estimation of uncertainties in section 5. At the end, section 6 , we discuss the reliability of the technique.

aFor the full authorlist see Appendix "Collaborations" in this volume.

This is an Open Access article distributed under the terms of the Creative Commons Attribution License 2.0, which permits unrestricted use, distribution, and reproduction in any medium, provided the original work is properly cited. 


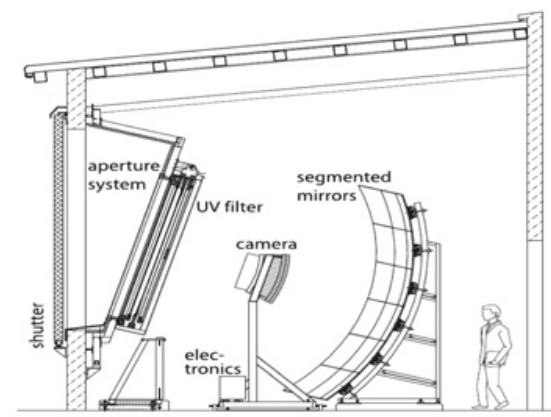

(a) Schematic view.

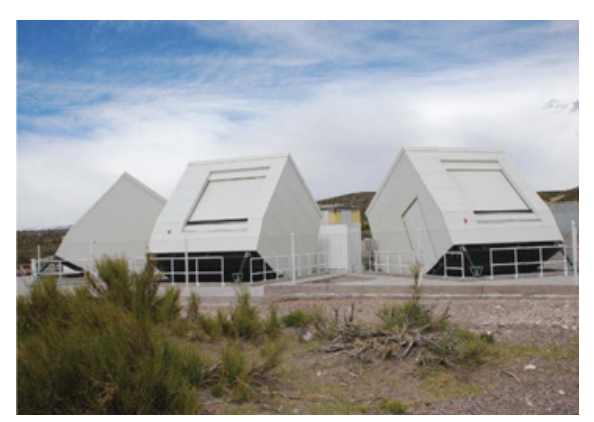

(b) HEAT.

Figure 1. Fluorescence telescopes.

\section{THE FLUORESCENCE TELESCOPE}

The Pierre Auger Observatory runs 27 fluorescence telescopes as illustrated in figure 1a. Four buildings house six telescopes each located on the edge of the array of surface detectors. Three extension telescopes named HEAT ${ }^{1}$ are installed on pivot-mounted enclosures that can be tilted as shown in figure $1 \mathrm{~b}$.

Each telescope covers $30^{\circ}$ in azimuth and $30^{\circ}$ in elevation. The standard telescopes cover from 2 to 32 degrees in elevation. The HEAT telescopes cover from 30 to 60 degrees in elevation. The HEAT extension was developed to study showers with energy between $10^{17}$ and $10^{18} \mathrm{eV}$.

The telescopes are equipped with Schmidt optics consisting of a segmented mirror of $11 \mathrm{~m}^{2}$, with radius of curvature of $3.4 \mathrm{~m}$ and a $2.2 \mathrm{~m}$ diameter diaphragm. The opening of the telescope is covered by a filter that selects the wavelength range corresponding to the emission of fluorescent light by nitrogen molecules in air (300-400 nm). After going through this sophisticated experimental arrangement, light produced by an air shower is focused onto a camera containing 440 photomultipliers.

Details about the fluorescence telescopes of the Pierre Auger Observatory have been described in reference [4].

\section{RECONSTRUCTION OF THE LONGITUDINAL PROFILE}

The $X_{\max }$ parameter is calculated as follows. First the arrival direction of the primary particle is determined using the time information of one tank and the signal in each pixel of the fluorescence telescope. Based on a time of flight technique, a combined fit of the hybrid information is able to reconstruct the arrival direction with median and $90 \%$ uncertainty of $0.35^{\circ}$ and $0.95^{\circ}$ respectively [5].

Knowing the direction of the shower axis, the light intensity measured by each photomultiplier in the telescope can be transformed into the energy deposited by the shower along the atmosphere. In this calculation the knowledge of the atmospheric extinction coefficients [6] and the fluorescence and Cherenkov yields must be used in each step.

The reconstruction procedure starts from the light intensity in the telescope and transforms it into the energy deposit at the shower axis. Figure $2 \mathrm{a}$ shows the number of photons as a function of time as estimated in the detector aperture for a particular event. It is also shown the estimated amount of

\footnotetext{
${ }^{1}$ HEAT $=$ High Elevation Angle Telescope.
} 


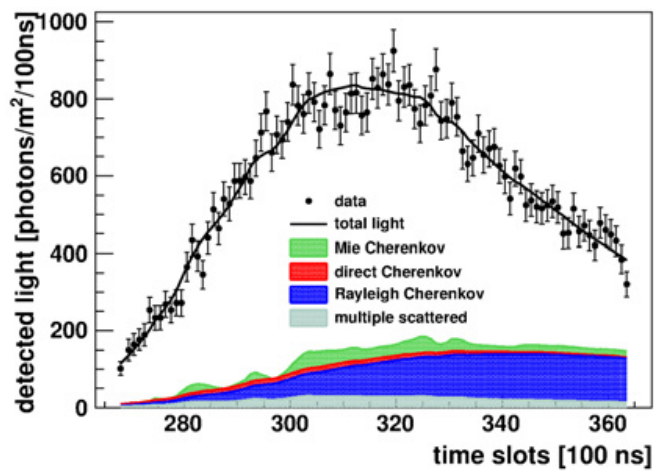

(a) Light at the aperture of the telescope.

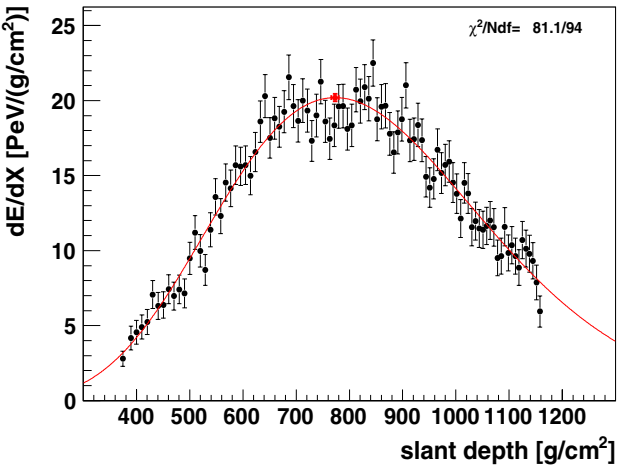

(b) Energy deposited into the atmosphere.

Figure 2. Longitudinal development of a particular shower chosen for illustration.

Cherenkov light. This information is transformed into the energy deposited into the atmosphere as shown in figure 2b. See ref. [7] for details. It is also shown a fit of a Gaisser-Hillas function [8]. One of the parameters fitted in the Gaisser-Hillas function is exactly $X_{\max }$ shown as the red dot in figure $2 \mathrm{~b}$.

\section{DATA SELECTION}

The data selection has two main purposes. Firstly we have to guarantee a good resolution of $X_{\max }$ and energy and secondly we need to eliminate a possible detection bias regarding primary particle types.

The list below shows the selection criteria for good quality events. They have been developed in a detailed study of our detectors:

1. Atmospheric Conditions: events are used if there are reliable measurements of the vertical optical depth of aerosols and they are smaller than 0.1 and if less than $25 \%$ of the sky is covered by clouds.

2. Time Synchronisation: showers with large apparent angular speed are rejected in order to minimise uncertainties in the time synchronisation between the FD and SD. Numerically we have excluded events with axis within $20^{\circ}$ from the telescope axis. This cut also rejects events with large fraction of direct Cerenkov light.

3. $X_{\max }$ in the Field-Of-View (FOV): studies have shown that the $X_{\max }$ and the energy can only be well reconstructed if the reconstructed $X_{\max }$ is in the FOV of the telescope. Therefore if only the rising or falling parts of an event are measured it is rejected.

4. Expected Statistical Uncertainty: the expected statistical uncertainty of the $X_{\max }$ reconstruction is calculated. We have chosen to use events in which the expected statistical uncertainty is smaller than $40 \mathrm{~g} / \mathrm{cm}^{2}$.

5. Profile Fit Quality: longitudinal profiles can be distorted by residual cloud contamination. Loose cuts on the profile fit quality (fit to a Gaisser-Hillas) are applied such that only events with $\chi^{2} / \mathrm{Ndf}<2.5$ are used in the analysis.

Some of these selection cuts and the intrinsic efficiency of the telescopes might change the probability detection of showers with large or small $X_{\max }$. Events with $X_{\max }$ outside the FOV are rejected. Deep or shallow showers might be rejected if the $X_{\max }$ is close to the limits of the FOV. The effect of this bias would under sample the $X_{\max }$ distribution. Figure 3 shows an example of the effect of this bias. This example was produced based on Monte Carlo simulation of the shower and telescope. Figure 3 shows two distributions, one distribution is shown as the black line and is labelled true distribution which was the $X_{\max }$ distribution generated by the Monte Carlo program. The other distribution shown as the red 


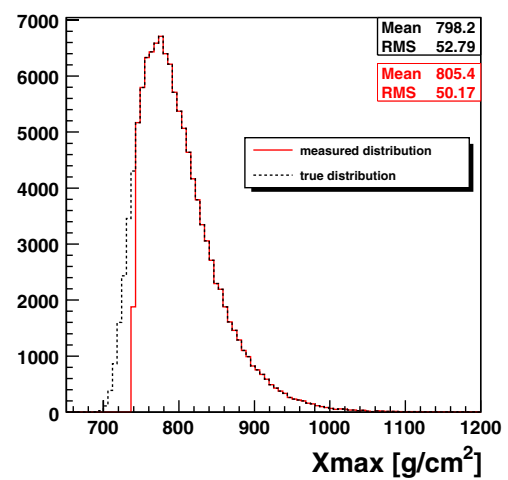

Figure 3. Illustration of the truncation of the $X_{\max }$ distributions. See text.

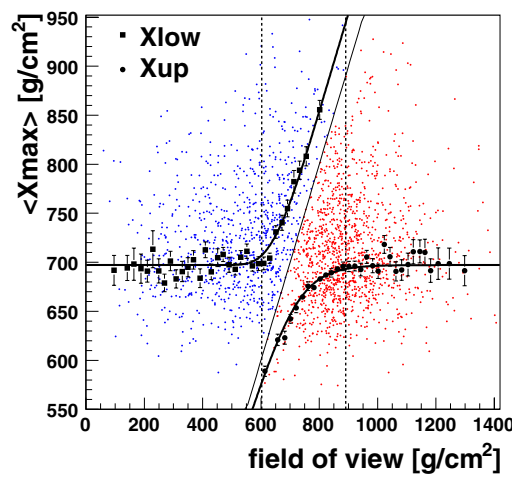

Figure 4. $X_{\max }$ as a function of FOV limits $\left.\left(10^{18.1}<E(\mathrm{eV})\right)<10^{18.2}\right)$.

line is labelled measured distribution and shows the reconstructed $X_{\max }$ distribution after data analysis and quality selection cuts. The bias in the $X_{\max }$ distribution is clear by the rejection of shallow $X_{\max }$ events. One of the consequences of this bias is that $\left\langle X_{\max }\right\rangle$ of the $X_{\max }$ distributions has changed from 798.2 to $805.4 \mathrm{~g} / \mathrm{cm}^{2}$.

We have studied this effect using the data. For each measured shower that survived the quality cuts, we can calculate $X_{\max }$ and the points where the shower crosses the FOV limits of the telescopes. Using this data we can study the dependence of $\left\langle X_{\max }\right\rangle$ as a function of the lower FOV limit (Xlow) and the upper FOV limit (Xup).

We would like to consider showers with Xlow and Xup which guarantee an unbiased data sample. Figure 4 shows the dependence of $\left\langle X_{\max }\right\rangle$ with Xlow and Xup for showers with energy $10^{18.1}<E<$ $10^{18.2} \mathrm{eV}$. Blue and red dots show the $X_{\max }$ versus Xlow and Xup respectively for each measured event. Black squares and circles show $\left\langle X_{\max }\right\rangle$ versus Xlow and Xup respectively. The dashed lines show the limits of Xlow and Xup for which $\left\langle X_{\max }\right\rangle$ does not change. The region of Xlow and Xup in which $\left\langle X_{\max }\right\rangle$ does not change defines the fiducial volume of the detector for which the $X_{\max }$ distribution can be measured with minimum bias. The position of the dashed line was chosen such that $\left\langle X_{\max }\right\rangle$ is within $5 \mathrm{~g} / \mathrm{cm}^{2}$ of the asymptotic value. The same analysis was repeated for all energy intervals and the corresponding value of Xlow and Xup was calculated as shown in Figure 5. 
UHECR 2012

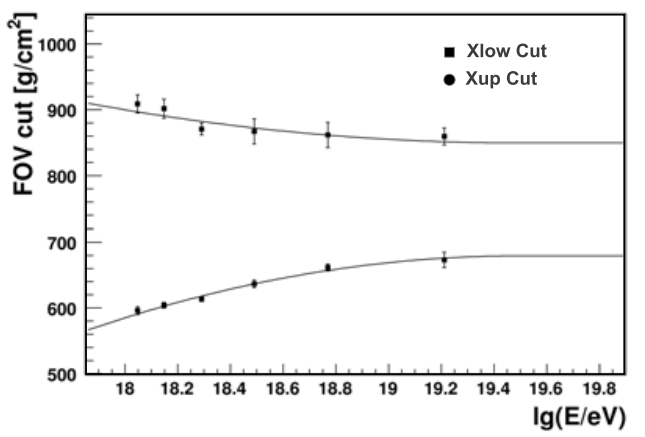

Figure 5. FOV cuts as a function of energy.

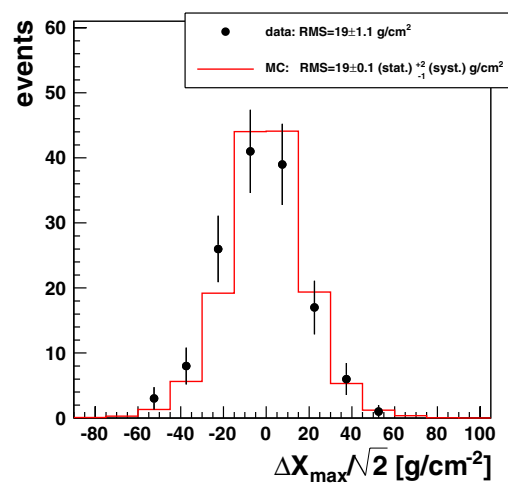

Figure 6. Comparison of $\mathrm{MC}$ and data.

\section{ESTIMATE OF UNCERTAINTIES}

The uncertainties of this analysis were estimated using a detailed Monte Carlo (MC) simulation of our detectors [9]. Before using the simulation we have certified its functionality by using events seen by two telescopes (stereo events). The redundant detection of some showers allows an independent reconstruction of the events including atmospheric uncertainties. The comparison of the two reconstructions gives the resolutions and uncertainties involved in the reconstruction procedure which can be compared to the MC calculations. Figure 6 shows the good agreement between the stereo events and the MC calculations.

Using simulations, the $X_{\max }$ resolution was shown to be at the $20 \mathrm{~g} / \mathrm{cm}^{2}$ level for energies above $10^{19} \mathrm{eV}$. Uncertainties of the calibration, atmospheric conditions, reconstruction and event selection (analysis) sum up to a systematic uncertainty smaller than $13 \mathrm{~g} / \mathrm{cm}^{2}$ for $\left(X_{\max }\right)$ and smaller than $6 \mathrm{~g} / \mathrm{cm}^{2}$ for the RMS. See Figure 7.

Figure 7a shows the contribution of each independent effect to the total systematic uncertainty of $\left\langle X_{\max }\right\rangle$. Figure $8 \mathrm{~b}$ shows the uncertainty divided in two labels: resolution and analysis. The resolution includes calibration and atmospheric effects. The resolution should be subtracted from the $\operatorname{RMS}\left(X_{\max }\right)$ calculated from the $X_{\max }$ distribution in order to compare the data to Monte Carlo predictions. 


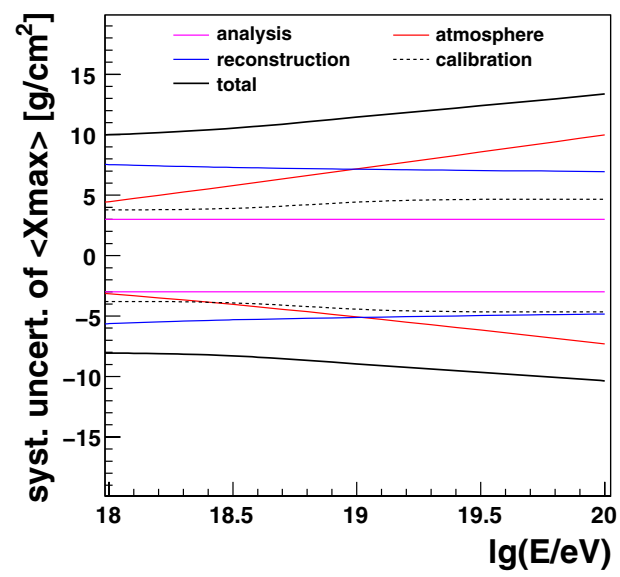

(a) $\left\langle X_{\max }\right\rangle$

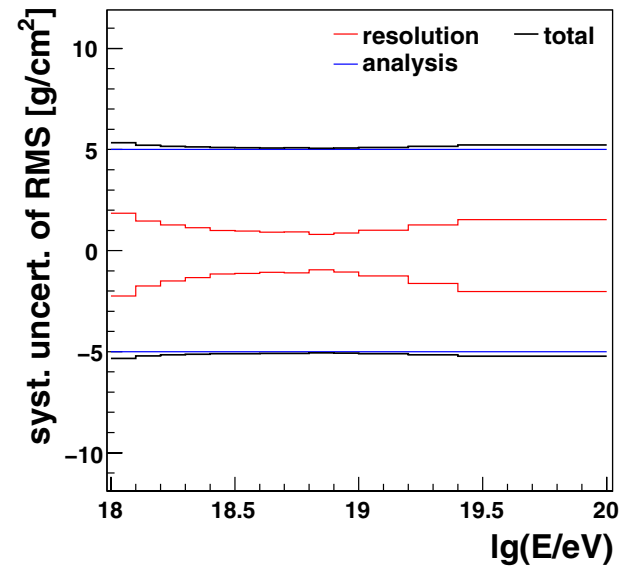

(b) $\operatorname{RMS}\left(X_{\max }\right)$

Figure 7. Systematic uncertainty of $X_{\max }$ and $\operatorname{RMS}\left(X_{\max }\right)$ as a function of energy.

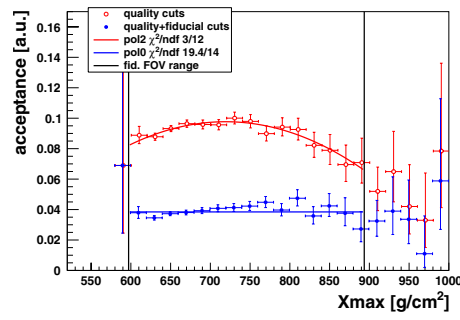

(a) $10^{18.1}<E<10^{18.2} \mathrm{eV}$.

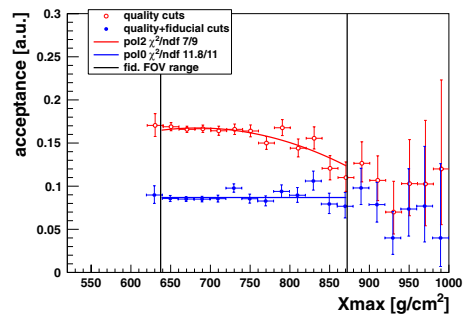

(b) $10^{18.5}<E<10^{18.6} \mathrm{eV}$.

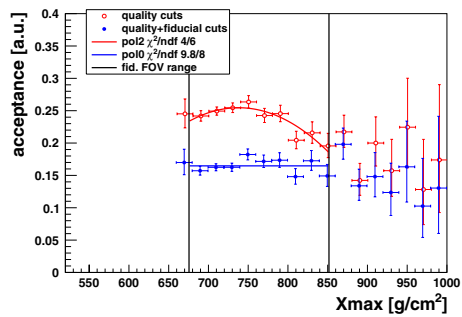

(c) $10^{19.2}<E<10^{19.4} \mathrm{eV}$.

Figure 8. Examples of the acceptance as a function of $X_{\max }$ for different energy bins.

\section{DISCUSSION}

We have described above the analysis procedure of the Pierre Auger Observatory used in order to select an unbiased sample of $X_{\max }$ distributions. Several tests have been applied to this analysis procedure. Below we enumerate some of them:

1. Independent data analysis: we developed an independent data analysis based on unfolding techniques which avoid the fiducial cuts to unbias the data;

2. We have studied the dependence of the results as a function of zenith angle. If the selected $X_{\max }$ sample has no bias, $\left\langle X_{\max }\right\rangle$ and $\operatorname{RMS}\left(X_{\max }\right)$ should not depend on zenith angle;

3. We have performed an analysis similar to that performed by the HiRes/TA collaborations [11, 12]. Instead of unbiasing the data we have biased the simulation predictions. Both ways can be compared using the conversion from $\left\langle X_{\max }\right\rangle$ to $\langle\ln A\rangle$.

These tests have been shown in this conference in the presentation of the Mass Composition WorkGroup [10]. The analysis procedure discussed here has successfully passed all tests: 1) the unfolding techniques resulted in the same evolution of $\left\langle X_{\max }\right\rangle$ and $\operatorname{RMS}\left(X_{\max }\right)$ with energy; 2) the unbiased $X_{\max }$ distribution has $\left\langle X_{\max }\right\rangle$ and $\operatorname{RMS}\left(X_{\max }\right)$ independent of zenith angle; 3$)$ the analysis similar to that performed by the HiRes/TA collaborations resulted in an equivalent dependence of $\langle\ln A\rangle$ with energy. 


\section{UHECR 2012}

The analysis procedure was developed in order to have an acceptance independent of $X_{\max }$. This guarantees an unbiased $X_{\max }$ distribution. Figure 8 shows the acceptance as a function of $X_{\max }$ with and without fiducial volume cuts. Without the cuts the acceptance is not constant and with the cuts it is flat within the used limits (Xlow and Xup) shown as black vertical lines.

The analysis and tests described in this article provide further details of the methods implemented to measure the energy dependence of $\left\langle X_{\max }\right\rangle$ and $\operatorname{RMS}\left(X_{\max }\right)[1]$ and the proton-air cross section [2, 3] with the Pierre Auger Observatory.

\section{References}

[1] The Pierre Auger Collaboration, Phys. Rev. Lett. 104 (2010) 091101

[2] The Pierre Auger Collaboration, Phys. Rev. Lett. (in press)

[3] R. Ulrich for the Pierre Auger Collaboration, these proceedings

[4] The Pierre Auger Collaboration, NIM A 620 (2010) 227-251

[5] The Pierre Auger Collaboration, 30 ${ }^{\text {th }}$ Proc. ICRC, Mexico 2007

[6] The Pierre Auger Collaboration, Astroparticle Physics 33 (2010) 108-129

[7] M. Unger et al., Nucl. Instrum. Meth. A 588 (2008) 433

[8] T. K. Gaisser and A. M. Hillas, Proc. 15th ICRC 8 (1977) 353-356, EA-88

[9] S. Argiro et al., NIM A 580 (2007) 1458

[10] J. Bellido et al., http://2012.uhecr.org

[11] R. Abbasi et al. HiRes Collaboration, Phys. Rev. Lett. 104 (2010) 161101

[12] C. Jui et al. TA Collaboration, Proc. APS DPF Meeting arXiv:1110.0133 